\title{
Low-magnitude whole body vibration does not affect bone mass but does affect weight in ovariectomized rats
}

\author{
Olav P. van der Jagt • Jacqueline C. van der Linden • \\ Jan H. Waarsing • Jan A. N. Verhaar • \\ Harrie Weinans
}

Received: 29 January 2011/ Accepted: 8 June 2011/Published online: 20 July 2011

(C) The Japanese Society for Bone and Mineral Research and Springer 2011

\begin{abstract}
Mechanical loading has stimulating effects on bone architecture, which can potentially be used as a therapy for osteoporosis. We investigated the skeletal changes in the tibia of ovariectomized rats during treatment with whole body vibration (WBV). Different low-magnitude WBV treatment protocols were tested in a pilot experiment using ovariectomized rats with loading schemes of $2 \times 8 \mathrm{~min} /$ day, 5 days/ week $(n=2$ rats per protocol). Bone volume and architecture were evaluated during a 10 week follow-up using in-vivo microcomputed tomography scanning. The loading protocol in which a $45 \mathrm{~Hz}$ sine wave was applied at $2 \mathrm{~Hz}$ with an acceleration of $0.5 \mathrm{~g}$ showed an anabolic effect on bone and was therefore further analyzed in two groups of animals ( $n=6$ each group) with WBV starting directly after or 3 weeks after ovariectomy and compared to a control (nonWBV) group at 0, 3, 6 and 10 weeks' follow-up. In the followup experiment the WBV stimulus did not significantly affect trabecular volume fraction or cortical bone volume in any of the treatment groups during the 10 week follow-up. WBV did reduce weight gain that was induced as a consequence of ovariectomy. We could not demonstrate any significant effects of WBV on bone loss as a consequence of ovariectomy in rats; however, the weight gain that normally results after ovariectomy was partly prevented. Treatment with WBV was not able to prevent bone loss during induced osteoporosis.
\end{abstract}

Keywords Osteoporosis - Whole body vibration . Rat $\cdot$ Micro-CT scanning $\cdot$ Weight management

O. P. van der Jagt $(\bowtie)$. J. C. van der Linden .

J. H. Waarsing - J. A. N. Verhaar · H. Weinans

Department of Orthopaedics, Erasmus MC, University Medical

Center Rotterdam, Dr. Molenwaterplein 40, Room 16.14,

PObox 2040, 3000 CA Rotterdam, The Netherlands

e-mail: o.vanderjagt@erasmusmc.nl

\section{Introduction}

Mechanical loading has potentially stimulating anabolic effects on bone architecture, which can be used as a therapy for osteoporosis, a disease characterized by low bone mass. Currently the standard treatment focuses on bone resorption inhibitors, which increase bone mineral density (BMD) and subsequently lower the risk for hip and vertebral fractures [1]; however, these pharmaceuticals require a frequent and (probably) lifelong intake with potential side-effects.

Whole body vibration (WBV) can be performed at different settings. In studies examining the effects on bone it is generally applied in a low-magnitude mode, resulting in small microstrains ( $<10$ microstrains). The osteogenic potential of WBV was demonstrated by a study in which the hind legs of sheep were subjected to mechanical vibration for 1 year $(30 \mathrm{~Hz}$ and an acceleration of $0.3 \mathrm{~g}$, for $20 \mathrm{~min} /$ day) resulting in a $32 \%$ higher trabecular bone volume fraction compared to non-treated controls [2]. In rodents it was found that osteoclastic activity decreased and bone formation rate increased after low-magnitude WBV [3, 4].

Few animal studies have examined the effect of WBV on osteoporosis specifically. In a longitudinal study it was found that ovariectomized rats that received WBV had a higher BMD in the femur and the tibia compared to nonvibrated controls after 5 weeks of WBV but not after 12 weeks of WBV [5]. Oxlund et al. [6] found that WBV at $45 \mathrm{~Hz}, 3.0 \mathrm{~g}$ for $30 \mathrm{~min} /$ day did affect periosteal bone formation rate, but vibrations of $17 \mathrm{~Hz}, 0.5 \mathrm{~g}$ and $30 \mathrm{~Hz}$, $1.5 \mathrm{~g}$ did not result in significant differences from control animals. In another study bone formation rates were largely affected when WBV at $90 \mathrm{~Hz}, 0.15 \mathrm{~g}$ was applied but not at $45 \mathrm{~Hz}$ [7]. These altered bone formation rates at $90 \mathrm{~Hz}$ did 
result in a higher volume fraction in the epiphysis, but not in the metaphysis.

In studies using ulnar or tibial bending it was shown that inclusion of rest periods between loading cycles of seconds, hours and also weeks further stimulated the bone formation rate [8-10]. It was also reported that when a noise signal that is on its own not osteogenic, is added to a low-magnitude vibration, bone formation rate increased almost fourfold [11]. These studies suggest that a continuous stimulus might decrease the mechanosensitivity of the skeleton and that that nonlinear characteristics in the loading regime enhance the potential anabolic effects of WBV.

To further elucidate the anabolic effect of whole body mechanical vibration on osteoporosis we investigated the skeletal changes in the tibia of ovariectomized rats during treatment with WBV. In vivo microcomputed tomography (micro-CT) scanning was used to evaluate skeletal changes. First we explored different WBV characteristics in a few animals to identify potential osteogenic stimuli. Subsequently we examined a successful stimulus in a higher number of animals to confirm the osteogenic effect.

\section{Materials and methods}

In total 38 female Wistar rats were purchased (Harlan, Boxmeer, The Netherlands). The animals were housed in pairs in the institute's animal facility with $12 \mathrm{~h}$ light/dark regimen, in the presence of standard food pellets (CRM (P) from Special Diets Services, UK, with a gross energy of $15.01 \mathrm{MJ} / \mathrm{kg}$, containing $0.83 \%$ calcium and 3077.42 $\mathrm{IU} / \mathrm{kg}$ vitamin D) and water ad libitum. The study protocols were approved by the local Animal Experiments Committee (EUR 536 and 940) and were in accordance with Dutch law on animal experimentation.

To simulate osteoporosis a bilateral ovariectomy (OVX) was performed at 20 weeks of age. This was performed under sterile conditions using gas anesthesia (oxygen with
3\% isoflurane; Rhodia Organique Fine Ltd., Bristol, UK). Analgesics were given for 2 days: $0.05 \mathrm{mg} / \mathrm{kg} / 12 \mathrm{~h} \mathrm{bupr-}$ enorfine (Schering-Plough, Kenilworth, NJ, USA). During treatment with WBV the animals were moved from their housing cage to the cage that was connected to an oscillator. The oscillator was controlled with a custom-made computer program using Labview software (National Instruments, Austin, TX, USA). Using an accelerometer the accelerations of the cage were registered in real time in order to monitor the applied mechanical stimulus.

In the first phase of the study 9 signals were investigated with different characteristics (Table 1; Fig. 1). The effect of each signal was investigated in 2 animals per signal during a follow-up period of 10 weeks and compared to a non-vibration control group. The animals were subjected to WBV twice a day for $8 \mathrm{~min}$. Each stimulus consisted of a vertical acceleration (sinusoid waveform) with a superimposed noise signal [low-amplitude Gaussian quasi white noise $(0-50 \mathrm{~Hz})$ ] [11]. The examined mechanical vibrations varied in frequencies, accelerations and rest periods between the loading cycles as summarized in Table 1 and Fig. 1. WBV was started 2 days after OVX and in vivo micro-CT scans were performed at 0,3 and 10 weeks after OVX.

In this pilot phase one stimulus showed a positive effect and was analyzed in more detail in the follow-up experiment. This stimulus was a $45 \mathrm{~Hz}$ sinusoid waveform superimposed on $2 \mathrm{~Hz}, 0.5 \mathrm{~g}$ with $10 \mathrm{~s}$ on/off regime. Two groups of 6 animals were treated twice a day for $8 \mathrm{~min}, 5$ days per week. In one group WBV started immediately (day 2) after OVX and in the second group WBV started 3 weeks after OVX. A third group $(n=6)$ did not receive mechanical vibrations and served as a control. Bone microarchitecture was analyzed at $0,3,6$ and 10 weeks after OVX.

To evaluate the bone changes in the proximal tibia in vivo micro-CT scans were performed. Under gas anesthesia the hind leg of the rat, from the distal femur until the tibial diaphysis, was scanned with an 18-micron voxel size using an in vivo micro-CT scanner (Skyscan 1076 microtomograph, Kontich, Belgium) at a voltage of $60 \mathrm{kV}$, a
Table 1 Overview of experimental groups

Different characteristics of the nine protocols examined in the first phase of the experiment. The basic sinusoid waveform was superimposed by $0-50 \mathrm{~Hz}$ very low amplitude noise

\begin{tabular}{llll}
\hline No. & Frequency & Acceleration $(g)$ & Applied \\
\hline 1 & $2 \mathrm{~Hz}$ & 0.5 & $2 \times$ day, 5 days/week, every week \\
2 & $2 \mathrm{~Hz}$ & 2 & $2 \times$ day, 5 days/week, every week \\
3 & $45 \mathrm{~Hz}$ & 0.5 & $2 \times$ day, 5 days/week, every week \\
4 & $45 \mathrm{~Hz}$ & 2 & $2 \times$ day, 5 days/week, every week \\
5 & $45-90 \mathrm{~Hz}$ noise & 0.5 & $2 \times$ day, 5 days/week, every week \\
6 & $2 \mathrm{~Hz}$ & 0.5 & $2 \times$ day, 3 days/week, every week \\
7 & $2 \mathrm{~Hz}$ & 0.5 & $2 \times$ day, 5 days/week, 1 week applied, 1 week rest \\
8 & $2 \mathrm{~Hz}$ & 0.5 & $2 \times$ day, 5 days/week, 3 weeks applied/3weeks rest \\
9 & $45 \mathrm{~Hz}$ given at $2 \mathrm{~Hz}$ & 0.5 & $2 \times$ day, 5 days/week, every week \\
Control & Sham & Sham & $2 \times$ day, 5 days/week, every week \\
\hline
\end{tabular}




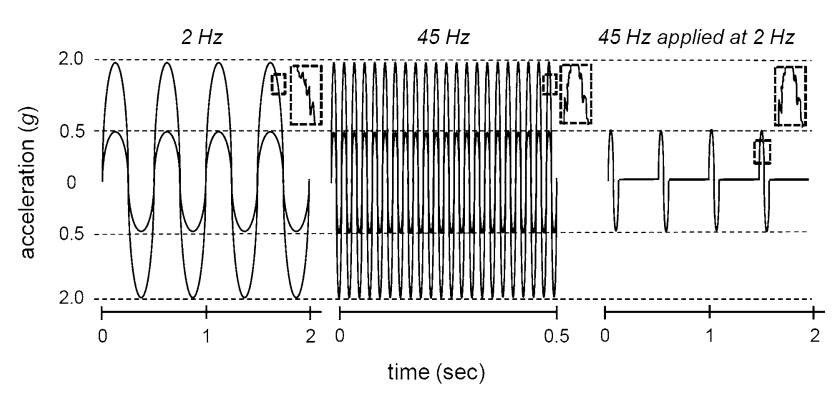

Fig. 1 Representation of different vibrations. Representation of vibration at 2,45 and a $45 \mathrm{~Hz}$ given at $2 \mathrm{~Hz}$, both 0.5 and $2.0 \mathrm{~g}$ acceleration. All vibrations were superimposed with $0-50 \mathrm{~Hz}$ noise signal (see inset)

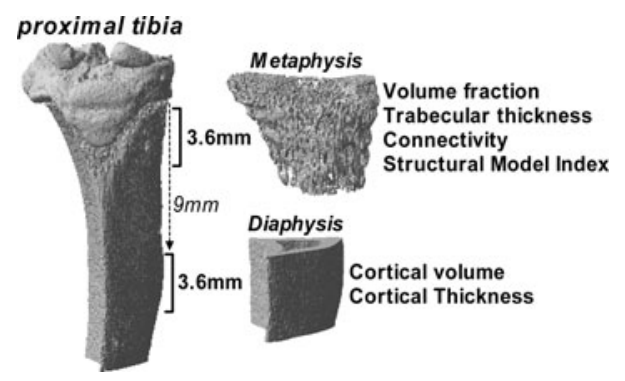

Fig. 2 Micro-CT scanning. 3D reconstruction of a micro-CT scan of the proximal tibia and proximal fibula. The analyzed regions of interest are indicated

current of $167 \mu \mathrm{A}$ and a $0.5 \mathrm{~mm}$ aluminum filter, over $196^{\circ}$ with a rotation step of $1^{\circ}$, taking 8 min per scan.

Using NRecon (NRecon software version 1.5, Skyscan) three-dimensional (3D) reconstructions of two regions of interest were made, one at the proximal metaphysis which mainly contains cancellous bone and another at the middiaphysis, which contains mainly cortical bone (Fig. 2). The reconstruction of the proximal metaphysis was selected manually starting just distally of the epiphysis and continuing distally until $3.6 \mathrm{~mm}$. The reconstruction of the diaphysis was defined by a region of $3.6 \mathrm{~mm}$ starting $9 \mathrm{~mm}$ distally from the epiphysis. Bony and non-bony structures were discriminated using a local threshold algorithm (software freely available) [12] resulting in binary datasets [13]. Cortical and trabecular bone were automatically separated using in-house software. Trabecular architecture of the proximal metaphysis was characterized by determining trabecular bone volume fraction (BV/TV), connectivity density (Conn/TV), structural model index (SMI) and mean 3D trabecular thickness (TbTh). Cortical architecture was assessed in the diaphysis and was characterized by cortical volume $(\mathrm{CtV})$ and cortical thickness $(\mathrm{CtTh})$.

For the follow-up experiment the differences between the means of each vibration group and the means of the control group were statically analyzed using ANOVA (GraphPad Software, San Diego, CA, USA).

\section{Results}

Experiment 1: pilot study

At the start of the experiment at 0 weeks the BV/TV was $34.4 \%$ (SD 4.4), TbTh was $117.7 \mu \mathrm{m}$ (SD 7.8), Conn/TV was 94.3 (SD 17.3), $\mathrm{CtV}$ was $15.2 \mu \mathrm{m}^{3}$ (SD 1.0), and $\mathrm{CtTh}$ was $644.9 \mu \mathrm{m}$ (SD 40.6).

During the experimental period the BV/TV declined in control animals to $23.2 \%$ (SD 3.7) at 3 weeks and to $14.2 \%$ (SD 3.7) at 10 weeks after OVX (Fig. 3a). Compared to the control group only one treatment showed a discriminative pattern of bone loss between 3 and 10 weeks (Fig. 3a). This specific treatment consisted of mechanical vibration of $45 \mathrm{~Hz}$ given at $2 \mathrm{~Hz}$ with an acceleration of $0.5 \mathrm{~g}$ (protocol no. 9, Table 1 and Fig. 1). Although both animals seemed to respond to this stimulus the magnitude was different, given that one animal showed anabolic effects and the other a subtle decrease in bone loss compared to controls (Table 2). Neither the cortical changes of this treatment group nor of the other treatment groups were different from control animals not receiving mechanical vibration (Fig. 3b; Table 2).

During the experimental period the average weight gain during the 10 week follow-up was not different between vibrated and control rats, with $68.8 \mathrm{~g}$ (SD 17.0) and $71.0 \mathrm{~g}$ (SD 4.2), respectively ( $p>0.05$ ).

\section{Experiment 2: follow-up study}

The BV/TV of control animals declined to $23.7 \%$ (SD 3.6) at 3 weeks, $16.3 \%$ (SD 2.6) at 6 weeks, and $12.0 \%$ (SD 2.4) at 10 weeks after OVX (Fig. 4). The CtV increased from $15.7 \mathrm{~mm}^{3}$ (SD 0.8) to 16.4 (SD 1.1), 17.1 (SD 1.0), and $17.2 \mu \mathrm{m}^{3}$ (SD 1.1) at 3, 6 and 10 weeks, respectively.

There was no difference between the animals that received WBV immediately after OVX and the control animals. In contrast to the exploratory phase, none of the six animals responded to the stimulus. Also, when 3 weeks of bone loss was allowed before the start of treatment, no effect from WBV was observed at an acceleration of $0.5 g$ (Fig. 4).

As a consequence of OVX the animals gained weight during follow-up; however, there was a difference between animals that received WBV and controls (Fig. 5a). Between 0 and 3 weeks no difference was observed, but animals that started WBV at 3 weeks lost weight between 3 and 6 weeks in contrast to controls, with $-1.3 \mathrm{~g}$ (SD 5.2) and $9.3 \mathrm{~g}$ (SD 4.7), respectively $(p<0.01)$. Between 6 and 10 weeks no significant difference was observed; however, the weight gain during the 10 week follow-up was different between controls and both WBV groups (Fig. 5b). The average weight gain of control rats was $88.5 \mathrm{~g}$ (SD 13.2) 
Fig. 3 Experiment 1: pilot study. Trabecular volume fraction (a) and cortical volume (b) in the proximal tibia of ovariectomized rats subjected to (sham)-WBV (mean with SD). Section of a $3 \mathrm{D}$ reconstruction of a control rat and a rat that received protocol no. 9 (c)

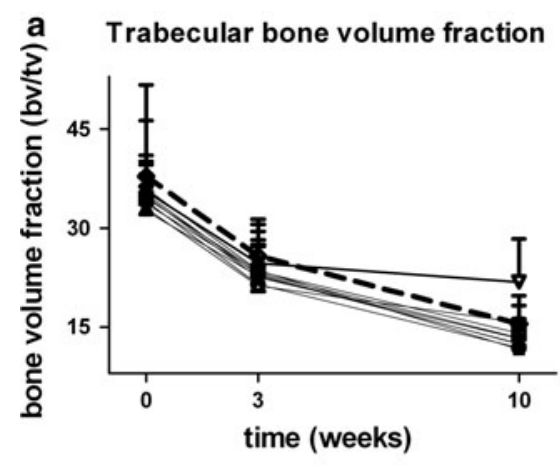

c

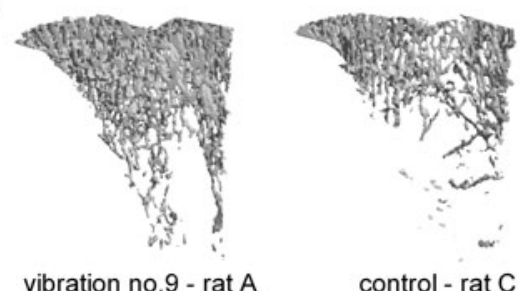

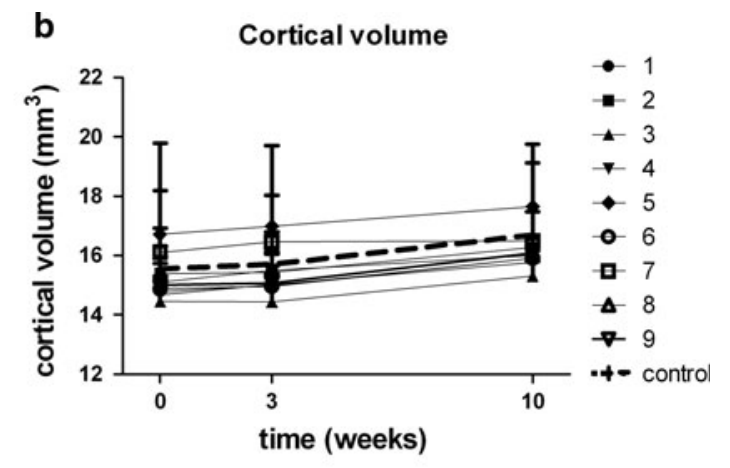

Table 2 Bone parameters in controls and $45 \mathrm{~Hz}$ at $2 \mathrm{~Hz} 0.5 \mathrm{~g}$ vibration

\begin{tabular}{|c|c|c|c|c|}
\hline & \multicolumn{2}{|l|}{ Week 3} & \multicolumn{2}{|l|}{ Week 10} \\
\hline & Control & Mechanical vibration & Control & Mechanical vibration \\
\hline BV/TV (\%) & $25.8(4.8)$ & $24.7(0.2)$ & $15.4(4.3)$ & $21.8(6.4)$ \\
\hline Trabecular thickness $(\mu \mathrm{m})$ & $108.2(2.0)$ & $105.1(1.0)$ & $112.1(0.8)$ & $114.6 *(1.4)$ \\
\hline SMI & $2.1(0.3)$ & $2.2(0.01)$ & $2.6(0.3)$ & $2.2(0.3)$ \\
\hline Connectivity density & $64.4(23.1)$ & $63.3(1.8)$ & $17.7(13.4)$ & $42.0(24.4)$ \\
\hline Cortical volume $\left(\mu \mathrm{m}^{3}\right)$ & $15.7(2.3)$ & $15.1(1.0)$ & $16.7(2.4)$ & $16.0(0.2)$ \\
\hline Cortical thickness $(\mu \mathrm{m})$ & $701.6(34.2)$ & $663.9(37.0)$ & $747(38.5)$ & $699(8.9)$ \\
\hline
\end{tabular}

Bone changes in the tibia of non-vibrated control rats and rats that received a $45 \mathrm{~Hz}$ vibration given at $2 \mathrm{~Hz}$ in the pilot study. WBV was started 2 days after OVX. Mean (SD), $n=2$ per group. Asterisk $\left(^{*}\right)$ indicates $p<0.05$ compared to control

Fig. 4 Experiment 2: follow-up study. Trabecular volume fraction (a) and cortical volume (b) in the proximal tibia of ovariectomized rats subjected to a $45 \mathrm{~Hz}$ vibration given at $2 \mathrm{~Hz}$, starting at week 0 or at week 3 after OVX (mean with SD)
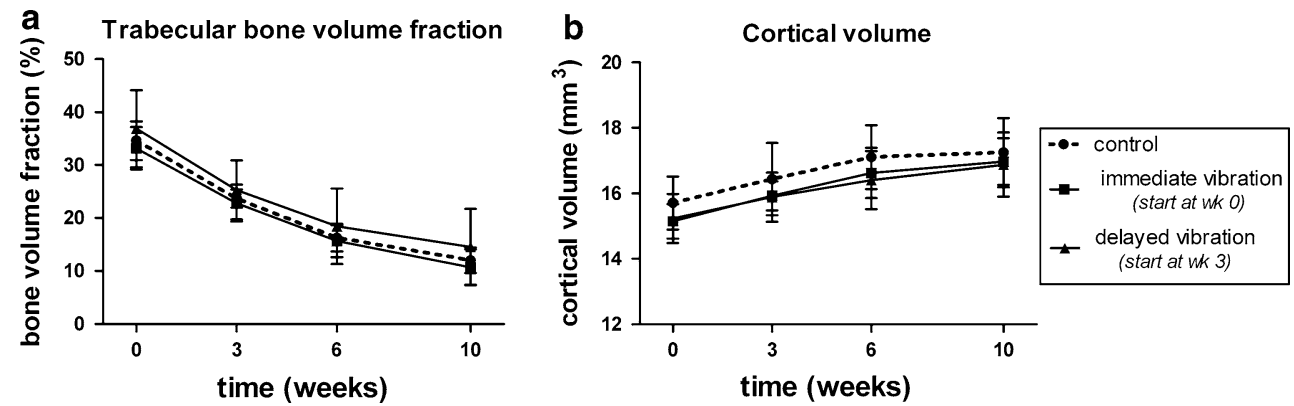

compared to $70.3 \mathrm{~g}$ (SD 6.4) and $62.7 \mathrm{~g}$ (SD 21.9) in the immediate vibration, and delayed vibration group ( $p=0.01$ and $p=0.03$, respectively).

\section{Discussion}

In the current study the effects of WBV were examined using in vivo micro-CT scanning allowing longitudinal follow-up of the bone microarchitecture in the proximal tibia of individual rats. In a pilot study, in which many different vibrations were examined, one stimulus affected the trabecular bone architecture; however, this effect could not be confirmed in the follow-up experiment. This data might suggest that some individual rats might be sensitive to a specific vibration, but it does not support the notion that WBV improves bone architecture as a benefit for osteoporotic patients in 

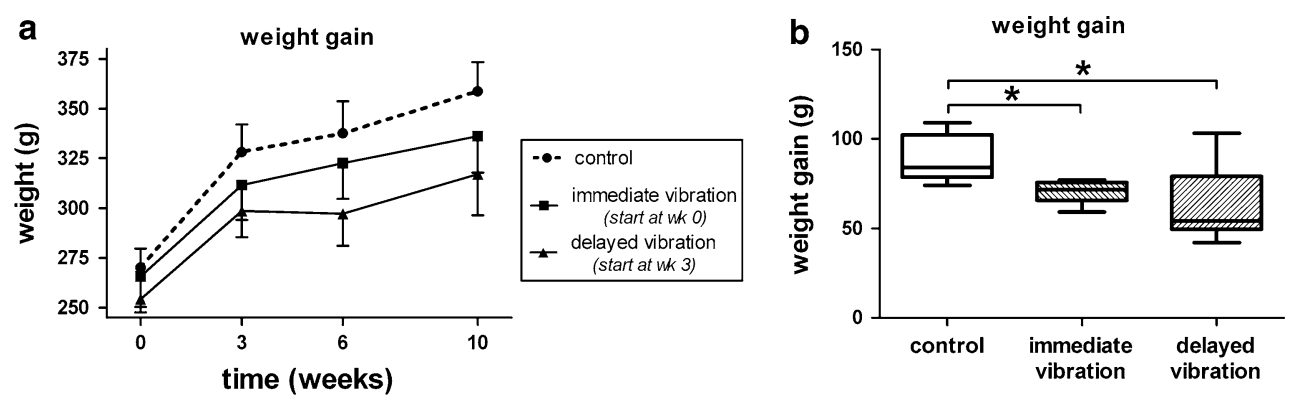

Fig. 5 Weight gain. Weight during follow-up (a) and 5-95\% whisker boxplot (of weight gain during the 10 week follow-up, Asterisk (*) indicates $p<0.05$ (b). The boxes represent the 5 to $95 \%$ confidence

general. However, WBV clearly reduced OVX-induced weight gain.

To date, positive findings of the effect of WBV on bone morphology are scarce. Rubin et al. found that sheep that received WBV for $20 \mathrm{~min} /$ day had $32 \%$ higher trabecular bone volume fraction than non-vibrated controls. These impressive results have never been reproduced in studies using rodents [2]. In mice it was shown that dynamic histomorphometric parameters like bone formation rate and osteoclastic resorption were clearly affected by WBV (45 Hz, $0.3 g, 15 \mathrm{~min} /$ day, 5 days/week), but cortical bone volume showed only a small increase and trabecular bone volume fraction was not affected $[3,4]$. In rats it was also observed that low-magnitude WBV $(45$ or $90 \mathrm{~Hz}, 0.15 \mathrm{~g}$, $10 \mathrm{~min} /$ day, 5 days/week) led to an increased bone formation rate in the metaphysis, while trabecular bone volume fraction did not increase [7].

Two other papers also report no effect of low-magnitude high-frequency WBV on microarchitectural parameters $[14,15]$. One study examined the effect of a $90 \mathrm{~Hz}$ sine wave with a maximum acceleration of $0.3 \mathrm{~g}$, applied 5 days/week in ovariectomized and sham-ovariectomized rats [14]. The other study used mice and was applied to a $90 \mathrm{~Hz}$ sine wave with a maximum acceleration of 0.3 or $1.0 \mathrm{~g}$ [15]. In contrast to these studies another study in which a $90 \mathrm{~Hz}$ WBV with an amplitude of $0.5 \mathrm{~mm}$ was applied to sham-ovariectomized rats did result in increased trabecular and cortical structural parameters [16].

Use of in vivo micro-CT scanning allows the detection of very small changes, because longitudinal data are collected. In our pilot experiment one stimulus seemed to reduce the OVX-induced bone loss; however, this effect could not be reproduced in our follow-up experiment. Given the data of the pilot study and the use of 6 animals, we would have been able to detect the large pilot effect $(>40 \%)$ with a power of close to 1 . Given the more robust data from the full-size experiment we would have been able to detect an effect of $11 \%$ (which is $2.6 \%$ difference in $\mathrm{BV} / \mathrm{TV}$ ) at a power of 0.8 and amplitude of 0.05 , which is intervals, the median is shown by the horizontal lines within the boxes, and the $I$ bars represent the minimum and maximum values

far less than the effect found during the pilot test $(>40 \%)$. Furthermore, the effect found at the pilot test was mainly caused by one animal that hardly lost any bone after treatment, suggesting that the effectiveness of a stimulus could be animal dependent. However, in our follow-up experiment none of the animals showed a reduction in bone loss that was different from the variation found in the control group.

To date, WBV in humans has shown contradictory effects. In a prospective study by Verschueren et al. it was shown that the BMD in the hip of postmenopausal women who received WBV for 24 weeks $(30-40 \mathrm{~Hz}, 2.3-5.1 \mathrm{~g}$ for a maximum of $30 \mathrm{~min} /$ day) was significantly higher than non-treated controls [17]. However, in another study with postmenopausal women, 6 months of WBV $(30-40 \mathrm{~Hz}$, $1.6-2.2 g, 12$ min three per week) did not influence BMD in the hip [18]. In a study by Rubin et al. in which postmenopausal women were included and in a study by Torvinen et al. of volunteers between 19 and 38 years, WBV did not affect the BMD at the hip, spine or distal radius after 12 and 8 months, respectively [19, 20]. WBV was also unable to enhance the BMD in the spine of osteoporotic patients treated with alendronate [21]. Bone serum markers were not influenced by WBV in any of these trials.

A shortcoming of the current paper is that no shamovariectomized animals were examined. There are some reports describing the interaction between the mechanosensitivity of the skeleton and the presence of estrogen and/ or estrogen-receptors $[22,23]$. Although this view is under a lot of debate [24] the study would have been stronger if the effect of the different WBVs were also examined in non-ovariectomized rats. However, in a recent paper using sham-ovariectomized and ovariectomized rats with loaded and unloaded hind legs it was demonstrated that the skeletal changes induced by estrogen loss were at other skeletal regions than the changes induced by loading and that the effects on bone due to estrogen or loading are therefore different entities [23]. Another limitation of the current study is that the strains induced by the vibrations were not 
measured. Since the characteristics of the vibration were at the same order of magnitude as the vibrations applied by others, we believe that the strains will also be at the same order of magnitude (between 1 and $2 \mu \varepsilon$ ) [7].

Since the effect of WBV on weight gain was unexpected in the current study we did not analyze which tissues were specifically affected; however, the effect of OVX on hyperphagia and subsequent gain in fat tissue has been extensively examined [25]. Currently, the effects of low-magnitude WBV on adipogenesis is receiving a lot of attention, since several reports have shown an inhibiting effect of low-magnitude WBV on adipogenesis and body fat accumulation [26-28]. These effects might be related to the differentiation of mesenchymal stem cells towards osteoblasts and adipocytes. It was found that the terminal differentiation of adipocyte progenitors is inhibited by loading, because PTH/PTHrP signalling pathways were affected [29]. This might also explain our findings of reduced weight gain in animals that received $\mathrm{WBV}$.

In conclusion the current study does not support the use of WBV as therapy for osteoporosis, but it was clearly effective at reducing OVX-induced weight gain.

Acknowledgments Funding was received from the Dutch Technology Foundation STW (RPG 6294).

\section{References}

1. Iwamoto J, Takeda T, Sato Y (2007) Effects of antifracture drugs in postmenopausal, male and glucocorticoid-induced osteoporosis-usefulness of alendronate and risedronate. Expert Opin Pharmacother 8:2743-2756

2. Rubin C, Turner AS, Bain S, Mallinckrodt C, McLeod K (2001) Anabolism. Low mechanical signals strengthen long bones. Nature 412:603-604

3. Xie L, Jacobson JM, Choi ES, Busa B, Donahue LR, Miller LM, Rubin CT, Judex S (2006) Low-level mechanical vibrations can influence bone resorption and bone formation in the growing skeleton. Bone 39:1059-1066

4. Xie L, Rubin C, Judex S (2008) Enhancement of the adolescent murine musculoskeletal system using low-level mechanical vibrations. J Appl Physiol 104:1056-1062

5. Flieger J, Karachalios T, Khaldi L, Raptou P, Lyritis G (1998) Mechanical stimulation in the form of vibration prevents postmenopausal bone loss in ovariectomized rats. Calcif Tissue Int 63:510-514

6. Oxlund BS, Ortoft G, Andreassen TT, Oxlund H (2003) Lowintensity, high-frequency vibration appears to prevent the decrease in strength of the femur and tibia associated with ovariectomy of adult rats. Bone 32:69-77

7. Judex S, Lei X, Han D, Rubin C (2007) Low-magnitude mechanical signals that stimulate bone formation in the ovariectomized rat are dependent on the applied frequency but not on the strain magnitude. J Biomech 40:1333-1339

8. Robling AG, Burr DB, Turner CH (2001) Recovery periods restore mechanosensitivity to dynamically loaded bone. J Exp Biol 204:3389-3399
9. Saxon LK, Robling AG, Alam I, Turner CH (2005) Mechanosensitivity of the rat skeleton decreases after a long period of loading, but is improved with time off. Bone 36:454-464

10. Srinivasan S, Weimer DA, Agans SC, Bain SD, Gross TS (2002) Low-magnitude mechanical loading becomes osteogenic when rest is inserted between each load cycle. J Bone Miner Res 17:1613-1620

11. Tanaka SM, Alam IM, Turner CH (2003) Stochastic resonance in osteogenic response to mechanical loading. FASEB J 17:313-314

12. The Erasmus Orthopaedic Research Laboratory, Erasmus MC, Rotterdam. http://www.erasmusmc.nl/47460/386156/Downloads. Accessed 10 July 2011

13. Waarsing JH, Day JS, Weinans H (2004) An improved segmentation method for in vivo microCT imaging. J Bone Miner Res 19:1640-1650

14. Brouwers JE, van Rietbergen B, Ito K, Huiskes R (2010) Effects of vibration treatment on tibial bone of ovariectomized rats analyzed by in vivo micro-CT. J Orthop Res 28:62-69

15. Lynch MA, Brodt MD, Silva MJ (2010) Skeletal effects of whole-body vibration in adult and aged mice. J Orthop Res 28:241-247

16. Sehmisch S, Galal R, Kolios L, Tezval M, Dullin C, Zimmer S, Stuermer KM, Stuermer EK (2009) Effects of low-magnitude, high-frequency mechanical stimulation in the rat osteopenia model. Osteoporos Int 20:1999-2008

17. Verschueren SM, Roelants M, Delecluse C, Swinnen S, Vanderschueren D, Boonen S (2004) Effect of 6-month whole body vibration training on hip density, muscle strength, and postural control in postmenopausal women: a randomized controlled pilot study. J Bone Miner Res 19:352-359

18. Verschueren SM, Bogaerts A, Delecluse C, Claessens AL, Haentjens P, Vanderschueren D, Boonen S (2011) The effects of whole-body vibration training and vitamin $\mathrm{D}$ supplementation on muscle strength, muscle mass, and bone density in institutionalized elderly women: a 6-month randomized, controlled trial. J Bone Miner Res 26:42-49

19. Rubin C, Recker R, Cullen D, Ryaby J, McCabe J, McLeod K (2004) Prevention of postmenopausal bone loss by a low-magnitude, high-frequency mechanical stimuli: a clinical trial assessing compliance, efficacy, and safety. J Bone Miner Res 19:343-351

20. Torvinen S, Kannus P, Sievanen H, Jarvinen TA, Pasanen M, Kontulainen S, Nenonen A, Jarvinen TL, Paakkala T, Jarvinen M, Vuori I (2003) Effect of 8-month vertical whole body vibration on bone, muscle performance, and body balance: a randomized controlled study. J Bone Miner Res 18:876-884

21. Iwamoto J, Takeda T, Sato Y, Uzawa M (2005) Effect of wholebody vibration exercise on lumbar bone mineral density, bone turnover, and chronic back pain in post-menopausal osteoporotic women treated with alendronate. Aging Clin Exp Res $17: 157-163$

22. Lanyon L, Skerry T (2001) Postmenopausal osteoporosis as a failure of bone's adaptation to functional loading: a hypothesis. J Bone Miner Res 16:1937-1947

23. Pajamaki I, Sievanen H, Kannus P, Jokihaara J, Vuohelainen T, Jarvinen TL (2008) Skeletal effects of estrogen and mechanical loading are structurally distinct. Bone 43:748-757

24. Raisz LG, Seeman E (2001) Causes of age-related bone loss and bone fragility: an alternative view. J Bone Miner Res 16:1948-1952

25. Jiang JM, Sacco SM, Ward WE (2008) Ovariectomy-induced hyperphagia does not modulate bone mineral density or bone strength in rats. J Nutr 138:2106-2110

26. Rubin CT, Capilla E, Luu YK, Busa B, Crawford H, Nolan DJ, Mittal V, Rosen CJ, Pessin JE, Judex S (2007) Adipogenesis is inhibited by brief, daily exposure to high-frequency, extremely 
low-magnitude mechanical signals. Proc Natl Acad Sci USA 104:17879-17884

27. Sen B, Xie Z, Case N, Ma M, Rubin C, Rubin J (2008) Mechanical strain inhibits adipogenesis in mesenchymal stem cells by stimulating a durable beta-catenin signal. Endocrinology 149:6065-6075

28. Maddalozzo GF, Iwaniec UT, Turner RT, Rosen CJ, Widrick JJ (2008) Whole-body vibration slows the acquisition of fat in mature female rats. Int J Obes (Lond) 32:1348-1354
29. Menuki K, Mori T, Sakai A, Sakuma M, Okimoto N, Shimizu Y, Kunugita N, Nakamura T (2008) Climbing exercise enhances osteoblast differentiation and inhibits adipogenic differentiation with high expression of PTH/PTHrP receptor in bone marrow cells. Bone 43:613-620 